Article

\title{
Neutralization Assessments Reveal High Cardiothoracic Ratio and Old Age as Independent Predictors of Low Neutralizing Antibody Titers in Hemodialysis Patients Receiving a Single Dose of COVID-19 Vaccine
}

\author{
Chun-Yu Chen ${ }^{1,2}$ D, Kuan-Ting Liu ${ }^{3,4}$, Shin-Ru Shih ${ }^{3,5,6}$, Jung-Jr Ye ${ }^{7}$, Yih-Ting Chen ${ }^{1,2}$, Heng-Chih Pan ${ }^{1,2}$, \\ Heng-Jung Hsu ${ }^{1,2}$, Chiao-Yin Sun ${ }^{1,2}$, Chin-Chan Lee ${ }^{1,2}$, Chun-Ying Wu ${ }^{8}$, Chi-Chun Lai ${ }^{2,9}$ and I-Wen Wu ${ }^{1,2, *}$
}

check for

updates

Citation: Chen, C.-Y.; Liu, K.-T.; Shih, S.-R.; Ye, J.-J.; Chen, Y.-T.; Pan, H.-C.; Hsu, H.-J.; Sun, C.-Y.; Lee, C.-C.; Wu, C.-Y.; et al. Neutralization Assessments Reveal High Cardiothoracic Ratio and Old Age as Independent Predictors of Low Neutralizing Antibody Titers in Hemodialysis Patients Receiving a Single Dose of COVID-19 Vaccine. J. Pers. Med. 2022, 12, 68. https:// doi.org/10.3390/jpm12010068

Academic Editors: Panayiotis G. Vlachoyiannopoulos and Paraskevi Katsaounou

Received: 17 December 2021

Accepted: 5 January 2022

Published: 7 January 2022

Publisher's Note: MDPI stays neutral with regard to jurisdictional claims in published maps and institutional affiliations.

Copyright: (C) 2022 by the authors. Licensee MDPI, Basel, Switzerland. This article is an open access article distributed under the terms and conditions of the Creative Commons Attribution (CC BY) license (https:// creativecommons.org/licenses/by/ $4.0 /)$.
1 Department of Nephrology, Chang Gung Memorial Hospital, Keelung 204, Taiwan; shone@cgmh.org.tw (C.-Y.C.); b9402031@cgmh.org.tw (Y.-T.C.); balour@cgmh.org.tw (H.-C.P.); r5267@cgmh.org.tw (H.-J.H.); sun3970@cgmh.org.tw (C.-Y.S.); leefang@cgmh.org.tw (C.-C.L.)

2 College of Medicine, Chang Gung University, Taoyuan 333, Taiwan; ccl404@cgmh.org.tw

3 Research Center for Emerging Viral Infections, College of Medicine, Chang Gung University, Taoyuan 333, Taiwan; jeff31602@gmail.com (K.-T.L.); srshih@mail.cgu.edu.tw (S.-R.S.)

4 Graduate Institute of Biomedical Science, College of Medicine, Chang Gung University, Taoyuan 333, Taiwan

5 Department of Medical Biotechnology and Laboratory Science, College of Medicine, Chang Gung University, Taoyuan 333, Taiwan

6 Graduate Institute of Medical Biotechnology and Laboratory Science, College of Medicine, Chang Gung University, Taoyuan 333, Taiwan

7 Department of Infectious Diseases, Chang Gung Memorial Hospital, Keelung 204, Taiwan; loyawise@cgmh.org.tw

8 Department of Laboratory Medicine, Chang Gung Memorial Hospital, Keelung 204, Taiwan; hla0861@cgmh.org.tw

9 Department of Ophthalmology, Chang Gung Memorial Hospital, Keelung 204, Taiwan

* Correspondence: a22066@cgmh.org.tw; Tel.: +886-2-24313131-6211

\begin{abstract}
Background: Data are lacking regarding predictors of quantification of neutralizing antibodies (nAbs) based on severe acute respiratory syndrome coronavirus 2 (SARS-CoV-2) 50\% neutralization titer $\left(\mathrm{NT}_{50}\right)$ after a single dose of COVID-19 vaccine in hemodialysis (HD) patients. Methods: This prospective single-center study enrolled $200 \mathrm{HD}$ patients and 82 healthy subjects to estimate antibodies against the SARS-CoV-2 viral spike protein 1 and receptor-binding domain after a first dose of a COVID-19 vaccine (ChAdOx1 or mRNA-1273), measured by enzyme-linked immunosorbent assay and applied spline-based generalized additive model regression analysis to predict $\mathrm{NT}_{50}$ converted to international units. Results: After the first dose of ChAdOx1, multiple linear regression showed that age $(p=0.011)$ and cardiothoracic ratio $(p=0.002)$ were negatively associated with $\mathrm{NT}_{50}$. Older age $(\mathrm{OR}=0.958, p=0.052)$ and higher cardiothoracic ratio $(\mathrm{OR}<0.001, p=0.037)$ could predict negative humoral response $\left(\mathrm{NT}_{50}<35.13 \mathrm{IU} / \mathrm{mL}\right)$. $\mathrm{NT}_{50}$ was lower in HD patients compared with healthy controls receiving ChAdOx1 (10.68 vs. $43.01 \mathrm{IU} / \mathrm{m}, p<0.001$ ) or mRNA-1273 (36.39 vs. $262.2 \mathrm{IU} / \mathrm{mL}, p<0.001)$. ChAdOx1 elicited lower GMTs than mRNA-1273 in the HD cohort (10.68 vs. $36.39 \mathrm{IU} / \mathrm{mL}, p<0.001$ ) and in healthy controls (43.01 vs. $262.22 \mathrm{IU} / \mathrm{mL}, p<0.001)$. Conclusion: High cardiothoracic ratio and old age could independently predict a decline in $\mathrm{nAb}$ titers in an HD cohort vaccinated with a single dose of ChAdOx1.
\end{abstract}

Keywords: COVID-19 vaccine; neutralizing antibodies; clinical response; hemodialysis; effectiveness

\section{Introduction}

The novel coronavirus, severe acute respiratory syndrome coronavirus 2 (SARS-CoV-2), has been declared a global emergency, affecting 263 million people worldwide, and continues to engender a tremendous global burden [1]. Advanced age, diabetes mellitus, cancer, 
cardiovascular disease, chronic liver disease, hypertension, and smoking have been identified as risk factors linked to the incidence of coronavirus disease 2019 (COVID-19) [2-4]. Higher risks of mortality caused by COVID-19 are found in patients with older age, more comorbidities, and immune dysfunction $[3,5]$.

Once patients have reached stage 5 of chronic kidney disease (estimated glomerular filtration rate $<15 \mathrm{~mL} / \mathrm{min} / 1.73 \mathrm{~m}^{2}$ ), in combination with signs and symptoms owing to uremia-including uremic bleeding, protein-energy wasting, fluid overload, pericarditis, refractory metabolic acidosis, or hyperkalemia-renal replacement therapy (RRT) should be administered. Hemodialysis (HD) is the most prevalent method of RRT in Taiwan [6]; however, common acute complications regarding HD deserve our attention, such as intradialytic hypotension, cramps, chest pain, and vascular access infection [7]. Acute kidney injury requiring RRT frequently occurs in severe COVID-19 [8]. Furthermore, maintenance HD patients are also more vulnerable to COVID-19, because of greater age, coexistence of comorbidities, and immune-dysregulated status on account of uremia, comorbidities, dialysis-procedure-related biocompatibility, and microinflammation $[9,10]$.

In-center HD patients are an extremely vulnerable group; they are immunocompromised, require lifelong attendance at crowded dialysis facilities, and often have substantially attenuated vaccine response. The incidence rate of COVID-19 was approximately 7.7\%and the overall mortality rate could have been as high as $22.4 \%$-in the HD population during the first wave of the global pandemic [11]. Therefore, proactive infection-preventive measures should be adopted, and extensive vaccination is needed in this fragile population.

COVID-19 vaccination has been proven to reduce the incidence rate, lower the morbidity and mortality risk, and remain effective even in patients with immunocompromised status. Although the immunogenicity of COVID-19 vaccines seems to be attenuated in immunocompromised subjects in comparison to the general population, the potential for severe COVID-19 in this population offsets the uncertainties [12-14]. The cellular response and humoral response in HD patients were reported to be inferior to those of the general population. Neutralizing antibody $(\mathrm{nAb})$ responses to the adenoviral vector vaccine ChAdOx1 (Oxford-AstraZeneca) were tempered in HD patients, and one-third of patients receiving the attenuated adenovirus Ad26.COV2.S vaccine (Johnson \& Johnson) failed to seroconvert even 28-60 days post-vaccination [15,16]. The phenomenon of reduced humoral response was also found in uremic patients and kidney allograft recipients who were vaccinated with a first dose of the mRNA vaccine BNT162b2 (Pfizer/BioNTech) [17,18].

Taiwan has attained efficacious containment of SARS-CoV-2 transmission, keeping up to 253 days of zero domestic confirmed cases since the start of the devastating worldwide pandemic in 2020. However, a second wave of the COVID-19 pandemic has emerged in northern Taiwan since 14 May 2021, with multiple clusters of transmission, and has spread into other regions of the country, leading to 753 deaths within two months [19]. The overall coverage rate of the first dose was only $1.5 \%$ during the time of community outbreak, when vaccine supply was extremely lacking. The process of national general vaccination lags behind most western countries undergoing more severe epidemics. The first phase of Taiwan's vaccination program mainly used imported ChAdOx1 (Oxford-AstraZeneca), and the national vaccination policy has prioritized inoculation in the elderly, severely ill, and immunocompromised populations. Because of several clustered infection events in HD facilities in northern Taiwan, contributing to grave mortalities, HD patients were listed as priority targets for vaccination in the middle of June 2021 [20]. Vaccination was also vigorously suggested by the International Society of Nephrology, the American Society of Nephrology, the European Dialysis and Transplant Association, and the Taiwan Society of Nephology [21-25].

As Taiwan has the highest prevalence of end-stage renal disease (ESRD) worldwide, effective vaccination to prevent COVID-19 infection in this vulnerable population remains crucial. However, the effectiveness of vaccination in ESRD patients, and how their immune responses differ from those of the general population, is not completely understood. For the tempered post-vaccination humoral response in uremic patients, an accurate and safe 
method to rapidly quantify the nAbs and to identify the most vulnerable HD patients remains crucial; those who have low titers of nAbs might be prioritized for booster shots. To fill this knowledge gap, we conducted an observational cohort study to compare the titers of nAbs between ESRD patients and subjects with normal renal function who received a single dose of a COVID-19 vaccine. We also delineated possible factors associated with responses to vaccination. This information may provide the necessary insight to design an effective vaccination program for ESRD patients [26].

\section{Materials and Methods}

\subsection{Study Design and Patient Characteristics}

This was an observational, prospective, single-center study to evaluate $\mathrm{nAb}$ response in HD patients after a single dose of COVID-19 vaccination carried out in Chang Gung Memorial Hospital Keelung Branch in Taiwan. Bedside COVID-19 inoculation policy for HD patients was promulgated by the national health authorities and the Taiwan Society of Nephrology, who uniformly provide the ChAdOx1 vaccine to HD facilities for injection. Patients older than 18 years and who had been on HD for $>3$ months were screened. Those patients with active malignancy, under immunosuppressive treatment, with a Child-Pugh liver cirrhosis score worse than A, with a history of prior infection with SARS-CoV-2, who had previously been vaccinated at another healthcare center, or who were unwilling to be vaccinated were excluded from $\mathrm{nAb}$ titer analysis. The same enrollment criteria were used for the healthy control group (normal renal function subjects) recruited from the community. Overall, 497 patients were receiving long-term maintenance HD in our hospital-affiliated dialysis center, and 356 of them received bedside ChAdOx1 vaccination, while 53 patients were vaccinated with mRNA-1273. A total of 200 vaccinated HD patients were enrolled into the study, comprising 174 patients receiving the ChAdOx1 vaccine and 26 patients receiving the mRNA-1273 vaccine (Figure 1a). The healthy control group comprised 82 individuals, including 67 ChAdOx1 subjects and 15 mRNA-1273 subjects (Figure 1b). Furthermore, SARS-CoV-2 rapid antigen tests were mandatory in all of our HD patients on a weekly basis, possibly helping to avoid potential false elevation of humoral response due to infection. Since national health policy recommends a 12-week interval before the second dose of vaccination, none of our patients have received the subsequent dose. Differences in $\mathrm{nAb}$ titers between HD patients and healthy controls, as well as between the two types of vaccine, were compared. In addition, clinical, laboratory, and dialysis-related parameters were recorded and correlated with serum titers of nAbs.

This study was performed in accordance with the Declaration of Helsinki, and was approved by the Ethics Committee of the Institutional Review Board at Chang Gung Memorial Hospital (IRB: 202001041A3C604 and 202100854B0A3). Written informed consent was obtained from all study participants.

\subsection{Sample Collection}

Blood samples were collected after overnight fasting, and were delivered immediately (within $4 \mathrm{~h}$ of collection) to the laboratory for biochemical analyses, complete blood counts, and antibody titers. Blood samples of HD patients were collected during a single HD session via venous chamber before dialysis treatment. A fraction of the samples were transferred to chilled tubes and centrifuged at $3000 \times g$ for $10 \mathrm{~min}$ at $4{ }^{\circ} \mathrm{C}$ to obtain the sera. Lipemic or hemolyzed sera were discarded. 


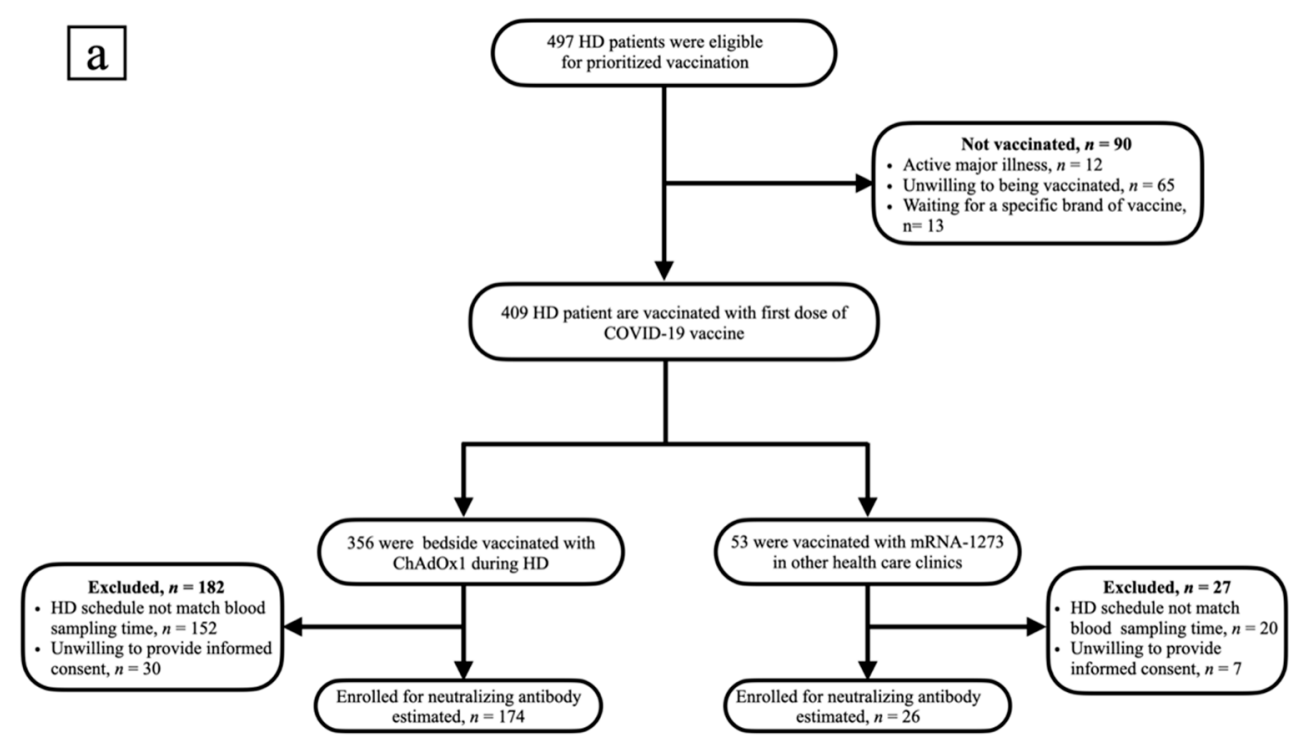

$\mathrm{b}$

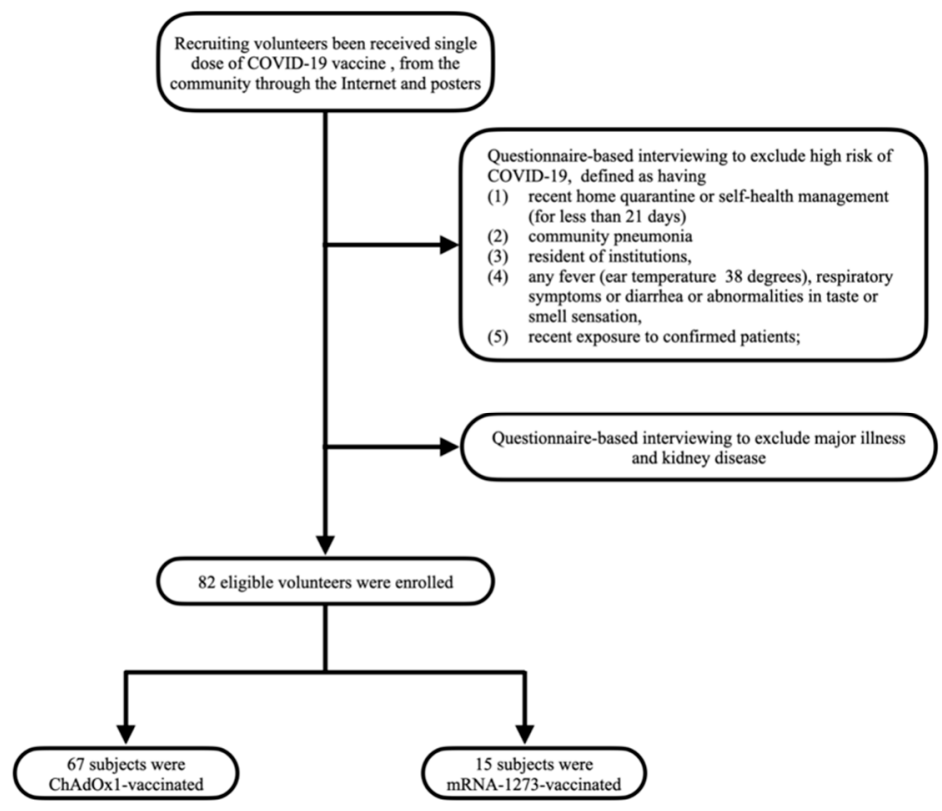

Figure 1. (a) Flow chart of patients on hemodialysis selected for neutralizing antibody measurement. (b) Flow chart of recruitment of healthy controls.

\subsection{Immunogenicity Response Assessment}

Immunogenicity was assessed by measuring $\mathrm{nAb}$ response on day 56 in HD patients and after a median of 30 (26-50) days in healthy control subjects after administering the first dose of COVID-19 vaccine, using the Formosa Biomedical Technology MeDiPro SARSCoV-2 Antibody ELISA assay, which detects antibodies against the SARS-CoV-2 viral spike protein 1 (S1) and receptor-binding domain (RBD). MeDiPro is a kit for quantifying nAbs using technology transferred from the Research Center for Emerging Viral Infections, Chang Gung University, and has been approved by the Taiwan Food and Drug Administration (No. 1106803303); the data for S1 and RBD fusion proteins can accurately predict the SARS-CoV-2 50\% neutralization titer $\left(\mathrm{NT}_{50}\right)$ under a two-variable generalized additive model and WHO international unit conversion (Supplementary Materials). According to the manufacturer, this test has $92.2 \%$ (95\% CI, 84.0-96.4\%) sensitivity and 93\% (95\% CI, 81.4-97.6\%) specificity. 


\subsection{Quantifying $n$ Abs by a Two-Variable Generalized Additive Model}

The MeDiPro SARS-CoV-2 Antibody ELISA kit was designed to detect SARS-CoV-2 nAbs in the serum, based on the binding affinity of S1 and RBD to antibodies. The RBD is the major binding site of nAbs. S1 covers the RBD and several other regions, which are also imperative for $\mathrm{nAb}$ binding. The assay combines each of the S1 and RBD ELISA unit (EU) values, and applies spline-based generalized additive model (GAM) regression analysis (using $\mathrm{S} 1$ and RBD as two predictors) to predict $\mathrm{NT}_{50}$ by combining multiple smooth functions.

\subsection{WHO International Standard Unit (IU) Conversion}

In order to facilitate the conversion of geometric mean titers (GMTs) of $\mathrm{NT}_{50}$ to international units, WHO international standard (IS) sera (20/130, 20/136, and 20/268) were obtained from the National Institute for Biological Standards and Control (NIBSC). IS sera were used to obtain $\mathrm{nAb}$ titers in IU/mL. The $\mathrm{NT}_{50}$ values for WHO IS sera were determined by a live virus microneutralization assay. Each standard serum sample was tested in duplicate, except for 20/130. To convert $\mathrm{NT}_{50}$ to $\mathrm{IU} / \mathrm{mL}$, a neutralizing assay was developed to calculate the GMTs of the NIBSC serum samples. Values of $<12.31 \mathrm{IU} / \mathrm{mL}$ $\left(\mathrm{NT}_{50}<2.56\right)$ were defined as negative humoral response, values between 12.31 and $35.13 \mathrm{IU} / \mathrm{mL}\left(2.56 \leq \mathrm{NT}_{50}<8\right)$ were defined as weakly positive humoral response, and values $>35.13 \mathrm{IU} / \mathrm{mL}\left(\mathrm{NT}_{50}>8\right)$ were defined as positive humoral response. For the cutoff values of $\mathrm{NT}_{50}(12.31 \mathrm{IU} / \mathrm{mL})$, we used the ELISA's limitation of detection (LOD). When we enter the value of the LOD into the model, we get the value of $12.31 \mathrm{IU} / \mathrm{mL}$. The cutoff value of $35.13 \mathrm{IU} / \mathrm{mL}$ comes from converting the lowest neutralizing titer (NT) to IU $/ \mathrm{mL}$. In our clinical practice, the serial dilutions of the virus neutralization assay started from 1:8, because any lower dilution of serum is toxic to the cells, and causes bias in determining the titer. As we input NT $=8$ to the model, we get the value of $35.13 \mathrm{IU} / \mathrm{mL}$.

\subsection{Statistical Analysis}

The demographics and clinical characteristics of 3 groups with different $\mathrm{NT}_{50}$ after a first dose of ChAdOx1 vaccination were compared by one-way analysis of variance (ANOVA) in order to compare the normally distributed variables, with values expressed as means with standard deviations, while categorical variables were tested using the chisquared test, and the nonparametric independent Kruskal-Wallis test was performed to compare the non-normally distributed variables, with values expressed as medians with interquartile ranges.

Simple linear regression and multiple regression were both applied to examine the association between independent variables and $\mathrm{NT}_{50}$ after the first dose of ChAdOx1 vaccination. The non-normally distributed variables were logarithmically transformed, and then were subjected to regression analysis. Model 1 multiple regression included all variables, while Model 2 multiple regression excluded anti-S1 and anti-RBD antibodies with age adjustment.

To assess the associations between positive humoral response and clinically important dialysis-related variables after the first dose of ChAdOx1 vaccination, the odds ratio to produce an adequate amount of $\mathrm{nAbs}$ (predicted $\mathrm{NT}_{50}>35.13 \mathrm{IU} / \mathrm{mL}$ ) was calculated by binary univariate and multivariable logistic regression, using the Enter method.

To compare the $\mathrm{nAb}$ responses between the two kinds of vaccine, age was compared using Student's $t$-test, gender and humoral response were compared using the chi-squared test, and non-normally distributed data of predicted $\mathrm{NT}_{50}$ and GMTs were compared by nonparametric independent Mann-Whitney $U$ test, with values expressed as medians with interquartile ranges.

The receiver operating characteristic (ROC) curves were plotted to predict the probability of a binary outcome, including age vs. positive humoral response and cardiothoracic ratio vs. positive humoral response. Differences were examined using the area under the receiver operating characteristic (AUROC) curve. 
Continuous variables were tested for normal distribution using skewness, kurtosis, and the Kolmogorov-Smirnov test. All statistical analyses were two-tailed, and a value of $p<0.05$ was considered statistically significant. Data were analyzed using the Statistical Package for the Social Sciences (SPSS, Inc., Chicago, IL, USA) version 26.0 for Mac. GraphPad Prism version 8 (GraphPad Software, Inc., San Diego, CA, USA) was used to calculate GMTs with 95\% confidence intervals (CIs), and to generate all of the graphs.

\section{Results}

\subsection{Study Design and Subject Characteristics}

A total of 200 vaccinated HD patients were enrolled, comprising 174 patients receiving the ChAdOx1 vaccine and 26 patients receiving the mRNA-1273 vaccine (Figure 1a). The healthy control group enrolled 82 individuals, including 67 ChAdOx1 subjects and 15 mRNA-1273 subjects (Figure 1b). The enrolled HD subjects had comparable age, albumin, Kt/V, and cardiothoracic ratio to non-enrolled HD subjects (Table S1). The mRNAvaccinated HD patients had a higher percentage of liver cirrhosis $(p=0.002)$ and a lower percentage of cardiovascular disease $(p=0.011)$ than ChAdOx1-vaccinated HD patients (Table S2). The Pearson's correlation coefficients ( $\mathrm{r}$ ) between age and $\mathrm{NT}_{50}$ were -0.3892 $(p<0.0001)$ in ChAdOx1-vaccinated subjects and $-0.3889(p=0.012)$ in mRNA-1273vaccinated subjects (Figure S1). Among the HD cohort, ChAdOx1-vaccinated patients had lower amounts of anti-S1 (2.28 vs. $3.89 \mathrm{EU} / \mathrm{mL}, p=0.001)$ and anti-RBD (2.14 vs. $3.16 \mathrm{EU} / \mathrm{mL}, p<0.001)$ antibodies than mRNA-1273-vaccinated patients. HD patients vaccinated with either ChAdOx1 or mRNA-1273 had similar age $(64.97 \pm 13.20$ vs. $68.51 \pm 10.35$, $p=0.192)$, percentage of diabetes (55.2\% vs. 57.7\%, $p=0.809)$, albumin (4.03 \pm 0.37 vs. $4.10 \pm 0.40, p=0.388), \mathrm{Kt} / \mathrm{V}(1.64 \pm 0.34$ vs. $1.76 \pm 0.26, p=0.079)$, and cardiothoracic ratio $(0.52 \pm 0.07$ vs. $0.52 \pm 0.07, p=0.928)$ (Table S2).

\section{2. $N_{50}$ and Clinical Characteristics of ChAdOx1-Vaccinated HD Patients}

HD patients who received a first dose of ChAdOx1 vaccination were stratified according to $\mathrm{NT}_{50}$ of $\mathrm{nAbs}$. The high-titer patients were more likely to have higher serum ALT and lower cardiothoracic ratio (Table 1). The baseline comorbidities and medications were similar among the three $\mathrm{NT}_{50}$ groups. Simple linear regression analysis for factors associated with $\mathrm{NT}_{50}$ showed that anti-S1 Abs and anti-RBD antibodies ( $\beta \pm \mathrm{SE}: 1.841 \pm 0.065$, $p<0.001$; and $\beta \pm$ SE: $2.250 \pm 0.0106, p<0.001$, respectively) were positively associated with $\mathrm{NT}_{50}$, while cardiothoracic ratio $(\beta \pm \mathrm{SE}:-2.026 \pm 0.729, p=0.006)$ was negatively associated with $\mathrm{NT}_{50}$ (Table 2). Moreover, we performed multiple linear regression analysis with a backward stepwise selection method to estimate factors associated with $\mathrm{NT}_{50}$. After adjusting all variables (Model 1$)$, age $(\beta \pm \mathrm{SE}:-0.003 \pm 0.001, p=0.046)$, hemoglobin $(\beta \pm \mathrm{SE}$ : $-0.136 \pm 0.056, p=0.017)$, uric acid $(\beta \pm \mathrm{SE}:-0.021 \pm 0.010, p=0.038)$, ferritin $(\beta \pm \mathrm{SE}$ : $-0.119 \pm 0.048, p=0.016)$, and $\mathrm{Ca} \times \mathrm{P}$ product $(\beta \pm \mathrm{SE}:-0.003 \pm 0.001, p=0.006)$ were significantly, independently, and negatively associated with $\mathrm{NT}_{50}$, while anti-Si antibodies $(\beta \pm$ SE: $1.490 \pm 0.128, p<0.001)$, anti-RBD antibodies $(\beta \pm$ SE: $0.589 \pm 0.165, p=0.001)$, red blood cells $(\beta \pm$ SE: $2.787 \pm 1.313, p=0.036)$, mean corpuscular volume $(\beta \pm$ SE: $0.019 \pm 0.008, p=0.014)$, and transferrin saturation $(\beta \pm \mathrm{SE}: 0.003 \pm 0.001, p=0.006)$ were independently positively associated with $\mathrm{NT}_{50}$. An additional multiple linear regression analysis was conducted with all variables, but excluding anti-S1 and anti-RBD antibodies (Model 2), and found that red blood cells ( $\beta \pm$ SE: $6.169 \pm 3.063, p=0.046)$ and mean corpuscular volume ( $\beta \pm$ SE: $0.042 \pm 0.018, p=0.022)$ were independently positively associated with $\mathrm{NT}_{50}$ for $\mathrm{nAbs}$, while age $(\beta \pm \mathrm{SE}:-0.009 \pm 0.003, p=0.011), \mathrm{K}(\beta \pm \mathrm{SE}$ : $-0.115 \pm 0.057, p=0.046)$, and cardiothoracic ratio $(\beta \pm \mathrm{SE}:-2.259 \pm 0.719, p=0.002)$ were independently negatively associated with $\mathrm{NT}_{50}$ (Table 2$)$. 
Table 1. Baseline characteristics of patients receiving a single dose of ChAdOx1 vaccine, stratified by $\mathrm{NT}_{50}$ titer.

\begin{tabular}{|c|c|c|c|c|}
\hline & $\begin{array}{c}\mathrm{NT}_{50}<12.31 \mathrm{IU} / \mathrm{mL} \\
(n=135)\end{array}$ & $\begin{array}{c}12.31 \leq \underset{N_{50} \text { Titer }<35.13}{(n=16)}\end{array}$ & $\begin{array}{c}\mathrm{NT}_{50} \text { Titer } \geq 35.13 \\
(n=23)\end{array}$ & $p$-Value \\
\hline Age, year & $65.67 \pm 12.76$ & $61.69 \pm 13.70$ & $60.70 \pm 14.63$ & $0.094 \&$ \\
\hline Male, $n(\%)$ & $72(53.3)$ & $8(50)$ & $14(60.9)$ & 0.754 \\
\hline \multicolumn{5}{|l|}{ Comorbidities, $n(\%)$} \\
\hline Diabetes & $74(54.8)$ & $9(56.3)$ & $13(56.5)$ & 0.984 \\
\hline Dyslipidemia & $49(36.3)$ & $8(50)$ & $10(43.5)$ & 0.494 \\
\hline Liver cirrhosis & $4(3)$ & $0(0)$ & $0(0)$ & 0.551 \\
\hline Cardiovascular disease & $49(36.3)$ & $7(43.8)$ & $8(34.8)$ & 0.824 \\
\hline \multicolumn{5}{|l|}{ Baseline medications, $n(\%)$} \\
\hline Immunosuppressants & $8(5.9)$ & $1(6.3)$ & $0(0)$ & 0.485 \\
\hline RAAS blockade & $60(44.4)$ & $4(25)$ & $11(47.8)$ & 0.294 \\
\hline$\beta$-blockers & $55(40.7)$ & $6(37.5)$ & $12(52.2)$ & 0.549 \\
\hline Statins & $45(33.3)$ & $7(43.8)$ & $10(43.5)$ & 0.500 \\
\hline Anti-S1 antibody (EU/mL) & $2.11(1.96-2.41)$ & $3.49(3.14-3.86)$ & $5.99(5.18-9.61)$ & $<0.001^{\$}$ \\
\hline Anti-RBD antibody (EU/mL) & $2.07(1.86-2.26)$ & $3.16(3.04-3.55)$ & $3.79(3.22-5.37)$ & $<0.001^{\$}$ \\
\hline Hemoglobin $(\mathrm{g} / \mathrm{dL})$ & $10.04 \pm 1.14$ & $10.23 \pm 1.55$ & $10.20 \pm 1.19$ & $0.561^{\&}$ \\
\hline WBC $(1000 / \mu \mathrm{L})$ & $5.90(4.70-7.50)$ & $7.10(5.80-7.85)$ & $6.30(5.30-7.80)$ & $0.183^{\$}$ \\
\hline Platelet $(1000 / \mu \mathrm{L})$ & $187.65 \pm 65.41$ & $240.56 \pm 86.20$ & $189.52 \pm 76.62$ & $0.905^{\&}$ \\
\hline Albumin $(\mathrm{g} / \mathrm{dL})$ & $4.025 \pm 0.38$ & $3.96 \pm 0.32$ & $4.09 \pm 0.32$ & $0.465^{\&}$ \\
\hline Cholesterol (mg/dL) & $154.85 \pm 37.23$ & $160.44 \pm 32.32$ & $147.91 \pm 35.72$ & $0.556^{\&}$ \\
\hline Triglyceride (mg/dL) & $114.00(78.00-173.50)$ & $152.50(111.00-304.25)$ & $128.00(77.00-164.00)$ & $0.160^{\$}$ \\
\hline $\operatorname{AST}(\mathrm{U} / \mathrm{L})$ & $16.0(13.0-20.5)$ & $18.50(14.00-25.25)$ & $17(14-21)$ & $0.380^{\$}$ \\
\hline $\operatorname{ALT}(\mathrm{U} / \mathrm{L})$ & $13(10-19)$ & $16.50(10.25-21.50)$ & $17(13-21)$ & $0.043 * \$$ \\
\hline $\operatorname{Alk-P}(\mathrm{U} / \mathrm{L})$ & $92(71-146)$ & $114.00(80.00-235.25)$ & $79(65-116)$ & $0.136^{\$}$ \\
\hline Total bilirubin (mg/dL) & $0.40(0.30-0.40)$ & $0.35(0.20-0.40)$ & $0.40(0.30-0.40)$ & $0.749^{\$}$ \\
\hline $\operatorname{Bun}(\mathrm{mg} / \mathrm{dL})$ & $69.97 \pm 22.94$ & $68.73 \pm 19.43$ & $67.99 \pm 12.97$ & $0.686^{\&}$ \\
\hline Creatinine (mg/dL) & $9.65 \pm 2.59$ & $9.22 \pm 3.14$ & $10.06 \pm 1.88$ & $0.475^{\&}$ \\
\hline Uric acid (mg/dL) & $6.28 \pm 1.95$ & $6.21 \pm 1.83$ & $6.18 \pm 1.84$ & $0.810^{\&}$ \\
\hline $\mathrm{Na}(\mathrm{meq} / \mathrm{L})$ & $138.14 \pm 3.08$ & $137.5 \pm 3.86$ & $138.43 \pm 2.73$ & $0.676^{\&}$ \\
\hline $\mathrm{K}(\mathrm{meq} / \mathrm{L})$ & $4.76 \pm 0.80$ & $4.77 \pm 0.78$ & $4.57 \pm 0.71$ & $0.291 \&$ \\
\hline $\mathrm{Ca}(\mathrm{mg} / \mathrm{dL})$ & $9.35 \pm 0.85$ & $9.39 \pm 0.78$ & $9.67 \pm 0.62$ & $0.081^{\&}$ \\
\hline $\mathrm{P}(\mathrm{mg} / \mathrm{dL})$ & $5.26 \pm 1.59$ & $5.41 \pm 1.95$ & $5.36 \pm 1.45$ & $0.772 \&$ \\
\hline C-reactive protein $(\mathrm{mg} / \mathrm{L})$ & $3.60(1.30-9.20)$ & $6.30(2.70-9.90)$ & $4.45(1.63-9.30)$ & $0.457 \$$ \\
\hline Urea reduction rate & $76.00(70.50-79.50)$ & $75.00(68.25-80.00)$ & $76(72-79)$ & $0.695^{\$}$ \\
\hline Kt/V (Daugirdes) & $0.65 \pm 0.34$ & $1.61 \pm 0.29$ & $1.60 \pm 0.35$ & 0.562 \& \\
\hline nPCR (g/kg/day) & $1.10 \pm 0.58$ & $1.00 \pm 0.28$ & $1.04 \pm 0.25$ & $0.624^{\&}$ \\
\hline TACurea & $41.69 \pm 14.14$ & $42.00 \pm 12.18$ & $39.72 \pm 9.97$ & $0.519^{\&}$ \\
\hline $\operatorname{Iron}(\mu \mathrm{g} / \mathrm{dL})$ & $65.00(50.50-89.00)$ & $55(46-81)$ & $78(54-103)$ & $0.280^{\$}$ \\
\hline Ferritin (ng/mL) & $439.00(234.00-709.50)$ & $338.50(171.50-516.00)$ & $524(120-600)$ & $0.256^{\$}$ \\
\hline TSAT (\%) & $34.02 \pm 13.68$ & $29.77 \pm 8.66$ & $37.82 \pm 19.72$ & $0.240^{\&}$ \\
\hline Cardiothoracic ratio & $0.53 \pm 0.05$ & $0.48 \pm 0.06$ & $0.48 \pm 0.07$ & $0.006^{*}$ \\
\hline $\mathrm{Ca} \times \mathrm{P}$ product & $49.38 \pm 16.95$ & $50.66 \pm 18.43$ & $52.21 \pm 16.01$ & 0.461 \\
\hline
\end{tabular}

Notes: Data are presented as mean \pm standard deviation or median (interquartile range). Abbreviations-RAAS: renin-angiotensin-aldosterone system; WBC: white blood cell count; AST: aspartate transaminase; ALT: alanine transaminase; Alk-P: alkaline phosphatase; Bun: blood urea nitrogen; Kt/V: a mathematical formula representing a dose of dialysis; nPCR: normalized protein catabolic rate; $\mathrm{NT}_{50}$ : predicted SARS-CoV-2 $50 \%$ neutralization titer, expressed in international units; TACurea: time-averaged urea concentration; TSAT: transferrin saturation. *: statistically significant. ${ }^{\$}$ : comparison among HD patients with different humoral responses by nonparametric independent Kruskal-Wallis test. \&: Comparison among HD patients with different humoral responses by one-way ANOVA. 
Table 2. $\beta$-coefficients between predicted $\mathrm{NT}_{50}$ and independent variables.

\begin{tabular}{|c|c|c|c|c|c|c|}
\hline & \multicolumn{2}{|c|}{ Simple Linear Regression } & \multicolumn{2}{|c|}{$\begin{array}{c}\text { Multiple Regression Analysis, } \\
\text { Model } 1\end{array}$} & \multicolumn{2}{|c|}{$\begin{array}{c}\text { Multiple Regression Analysis, Model } \\
2\end{array}$} \\
\hline & $\beta \pm \mathrm{SE}$ & $p$ & $\beta \pm S E$ & $p$ & $\beta \pm S E$ & $p$ \\
\hline Age & $-0.005 \pm 0.003$ & 0.075 & $-0.003 \pm 0.001$ & $0.046^{*}$ & $-0.009 \pm 0.003$ & $0.011 *$ \\
\hline Anti-S1 antibody ${ }^{\$}$ & $1.841 \pm 0.065$ & $<0.001 *$ & $1.490 \pm 0.128$ & $<0.001 *$ & - & - \\
\hline Anti-RBD antibody ${ }^{\$}$ & $2.250 \pm 0.0106$ & $<0.001 *$ & $0.589 \pm 0.165$ & $0.001 *$ & - & - \\
\hline Hemoglobin & $0.004 \pm 0.032$ & 0.890 & $-0.136 \pm 0.056$ & $0.017^{*}$ & $-0.251 \pm 0.130$ & 0.054 \\
\hline $\mathrm{RBC}^{\$}$ & $-0.225 \pm 0.623$ & 0.718 & $2.787 \pm 1.313$ & $0.036 *$ & $6.169 \pm 3.063$ & $0.046^{*}$ \\
\hline $\mathrm{MCV}$ & $0.004 \pm 0.005$ & 0.478 & $0.019 \pm 0.008$ & 0.014 * & $0.042 \pm 0.018$ & $0.022 *$ \\
\hline $\mathrm{WBC}^{\$}$ & $0.375 \pm 0.246$ & 0.129 & - & - & - & - \\
\hline Platelet & $0.001 \pm 0.001$ & 0.254 & - & - & - & - \\
\hline Albumin & $0.061 \pm 0.104$ & 0.560 & - & - & - & - \\
\hline $\mathrm{AST}^{\$}$ & $0.011 \pm 0.222$ & 0.959 & - & - & - & - \\
\hline $\mathrm{ALT}^{\$}$ & $0.308 \pm 0.167$ & 0.066 & - & - & $0.401 \pm 0.191$ & $0.038 *$ \\
\hline Alk-P ${ }^{\$}$ & $-0.120 \pm 0.137$ & 0.380 & - & - & - & - \\
\hline Bilirubin $^{\$}$ & $-0.047 \pm 0.252$ & 0.853 & - & - & - & - \\
\hline Cholesterol & $-0.001 \pm 0.001$ & 0.564 & - & - & - & - \\
\hline Triglyceride $^{\$}$ & $0.034 \pm 0.151$ & 0.822 & - & - & - & - \\
\hline Creatinine & $0.007 \pm 0.015$ & 0.628 & $0.016 \pm 0.009$ & 0.073 & - & - \\
\hline Uric acid & $0.004 \pm 0.020$ & 0.858 & $-0.021 \pm 0.010$ & $0.038 *$ & - & - \\
\hline $\mathrm{Na}$ & $0.006 \pm 0.012$ & 0.632 & - & - & - & - \\
\hline $\mathrm{K}$ & $-0.049 \pm 0.048$ & 0.312 & - & - & $-0.115 \pm 0.057$ & 0.046 * \\
\hline $\mathrm{Ca}$ & $0.083 \pm 0.046$ & 0.074 & - & - & - & - \\
\hline $\mathrm{P}$ & $0.024 \pm 0.024$ & 0.320 & - & - & - & - \\
\hline C-reactive protein $\$$ & $0.072 \pm 0.064$ & 0.262 & - & - & - & - \\
\hline $\mathrm{URR}^{\$}$ & $0.038 \pm 0.240$ & 0.875 & $-0.655 \pm 0.354$ & 0.067 & - & - \\
\hline $\mathrm{Kt} / \mathrm{V}$ & $-0.048 \pm 0.117$ & 0.680 & - & - & - & - \\
\hline nPCR & $-0.043 \pm 0.074$ & 0.559 & - & - & - & - \\
\hline TACurea & $-0.001 \pm 0.003$ & 0.860 & - & - & - & - \\
\hline Ferritin $\$$ & $-0.062 \pm 0.095$ & 0.516 & $-0.119 \pm 0.048$ & $0.016^{*}$ & - & - \\
\hline Iron $\$$ & $0.245 \pm 0.216$ & 0.258 & - & - & - & - \\
\hline TSAT & $0.002 \pm 0.003$ & 0.465 & $0.003 \pm 0.001$ & $0.006 *$ & - & - \\
\hline Intact-PTH & $-0.000 \pm 0.000$ & 0.503 & - & - & - & - \\
\hline Cardiothoracic ratio & $-2.026 \pm 0.729$ & $0.006^{*}$ & $-0.555 \pm 0.305$ & 0.071 & $-2.259 \pm 0.719$ & $0.002 *$ \\
\hline $\mathrm{Ca} \times \mathrm{P}$ product & $0.003 \pm 0.002$ & 0.158 & $-0.003 \pm 0.001$ & $0.006^{*}$ & - & - \\
\hline
\end{tabular}

A backward stepwise selection method was applied for multivariate analysis. Abbreviations-WBC: white blood cell count; AST: aspartate transaminase; ALT: alanine transaminase; Alk-P: alkaline phosphatase; Bun: blood urea nitrogen; $\mathrm{Kt} / \mathrm{V}$ : a mathematical formula representing a dose of dialysis; $\mathrm{NT}_{50}$ : SARS-CoV-2 $50 \%$ neutralization titer, expressed in international units; nPCR: normalized protein catabolic rate; TACurea: time-averaged urea concentration; TSAT: transferrin saturation. *: Statistically significant; ${ }^{\$}: \log _{10}$ transformed.

\subsection{Predictors Associated with Positive Humoral Response in HD Patients}

Binary univariate followed by multivariable logistic regression analyses were applied to evaluate the risk of several dialysis-related parameters associated with high $\mathrm{NT}_{50}$. We found that cardiothoracic ratio (OR $<0.001,95 \%$ CI: 0.000-0.556, $p=0.037)$ was independently inversely related to $\mathrm{NT}_{50}$ over $35.13 \mathrm{IU} / \mathrm{mL}$, and age (OR: 0.958, 95\% CI: 0.917-1.000, $p=0.052$ ) also showed a similar tendency (Table 3). Figure 2 shows a receiver operating characteristic curve illustrating the performance of younger age (AUC: $62 \%$ ), lower cardiothoracic ratio (AUC: 67\%), and the combination obtained by multiplying the two (AUC: $70 \%$ ) in predicting the development of $\mathrm{nAb}$ titers over $35.13 \mathrm{IU} / \mathrm{mL}$ after a single dose of ChAdOx1 vaccination (Figure 2). The results suggest that there might be an interaction between age and cardiothoracic ratio affecting immunogenicity. 
Table 3. Binary logistic regression of factors associated with positive humoral response $\left(\mathrm{NT}_{50}>35.13 \mathrm{IU} / \mathrm{mL}\right)$ to a single dose of ChAdOx1 vaccine.

\begin{tabular}{|c|c|c|c|c|c|c|}
\hline & \multicolumn{3}{|c|}{ Univariate } & \multicolumn{3}{|c|}{ Multivariable } \\
\hline & OR & $95 \%$ CI & $p$-Value & OR & $95 \%$ CI & $p$-Value \\
\hline Male & 0.724 & $0.296-1.775$ & 0.481 & 0.809 & $0.214-3.056$ & 0.755 \\
\hline Age & 0.975 & $0.944-1.007$ & 0.125 & 0.958 & 0.917-1.000 & 0.052 \\
\hline Diabetes & 0.939 & $0.388-2.274$ & 0.889 & 0.849 & $0.285-2.528$ & 0.849 \\
\hline Liver cirrhosis & $2.54 \times 10^{8}$ & 0 & 0.999 & $1.09 \times 10^{7}$ & 0 & 1.000 \\
\hline Cardiovascular disease & 1.105 & $0.441-2.772$ & 0.831 & 0.915 & $0.269-3.116$ & \\
\hline Immunosuppressants & 0 & 0 & 0.999 & $2.37 \times 10^{-9}$ & 0 & 0.999 \\
\hline RAAS blockade & 1.246 & $0.517-3.003$ & 0.624 & 1.132 & $0.348-3.677$ & 0.837 \\
\hline$\beta$-blockers & 1.610 & $0.667-3.882$ & 0.289 & 0.612 & $0.199-1.885$ & 0.392 \\
\hline Hemoglobin & 1.102 & $0.760-1.599$ & 0.608 & 0.773 & $0.161-3.721$ & 0.748 \\
\hline $\mathrm{RBC}$ & 0.993 & $0.440-2.239$ & 0.987 & 1.934 & $0.015-252.653$ & 0.791 \\
\hline MCV & 1.020 & $0.955-1.090$ & 0.560 & 1.078 & $0.853-1.364$ & 0.528 \\
\hline Albumin & 1.700 & $0.482-5.999$ & 0.410 & 0.852 & $0.119-6.097$ & 0.873 \\
\hline ALT & 1.009 & $0.981-1.039$ & 0.526 & 1.007 & $0.969-1.046$ & 0.720 \\
\hline $\mathrm{K}$ & 0.727 & $0.407-1.301$ & 0.283 & 0.625 & $0.273-1.432$ & 0.267 \\
\hline C-reactive protein & 0.945 & $0.499-1.789$ & 0.861 & 1.008 & $0.972-1.045$ & 0.670 \\
\hline $\mathrm{Kt} / \mathrm{V}$ & 0.700 & $0.190-2.570$ & 0.591 & 0.977 & $0.131-7.294$ & 0.982 \\
\hline nPCR & 0.776 & $0.226-2.663$ & 0.687 & 0.556 & $0.053-5.784$ & 0.623 \\
\hline TSAT & 1.019 & $0.991-1.047$ & 0.189 & 1.015 & $0.982-1.049$ & 0.390 \\
\hline Cardiaothoracic ratio & 0.005 & $0.000-0.307$ & 0.012 & $<0.0001$ & $0.000-0.556$ & 0.037 * \\
\hline $\mathrm{Ca} \times \mathrm{P}$ product & 1.009 & $0.984-1.035$ & 0.477 & 1.019 & 0.981-1.059 & 0.325 \\
\hline
\end{tabular}

Abbreviations-RAAS: renin-angiotensin-aldosterone system; RBC: red blood cell count; MCV: mean corpuscular volume; ALT: alanine transaminase; Kt/V: a mathematical formula representing a dose of dialysis; $\mathrm{nPCR}$ normalized protein catabolic rate; $\mathrm{NT}_{50}$ : SARS-CoV-2 50\% neutralization titer, expressed in international units; TSAT: transferrin saturation; *: Statistically significant.

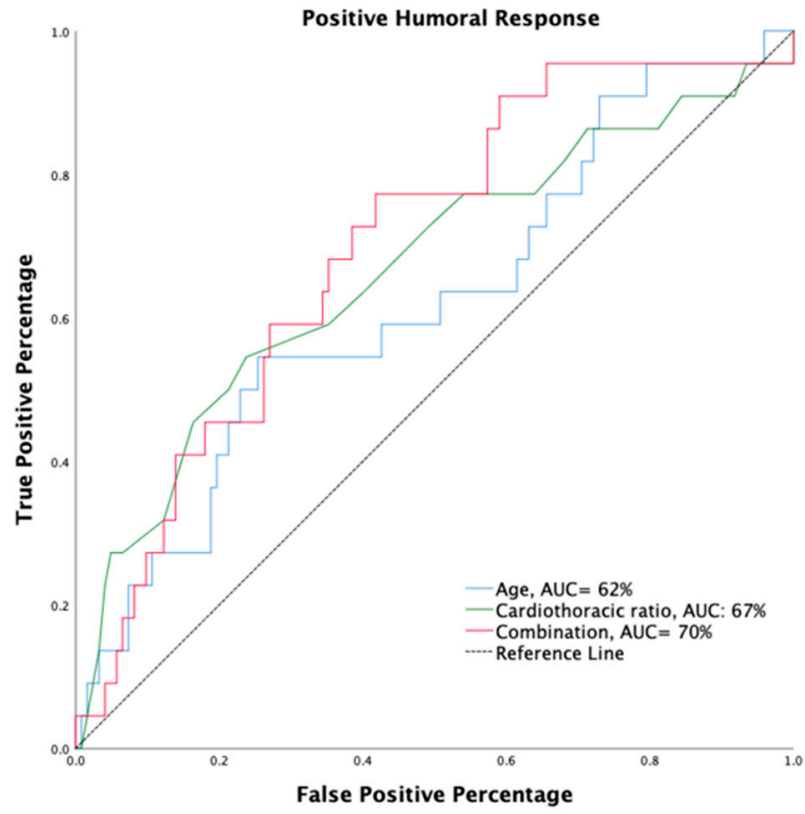

Figure 2. Receiver operating characteristic curve illustrating the performance of age, cardiothoracic ratio, and their combination in predicting the development of $\mathrm{NT}_{50}$ values over $35.13 \mathrm{IU} / \mathrm{mL}$ after a single dose of ChAdOx1 vaccination.

\subsection{Different Humoral Responses between HD Patients and Healthy Controls}

The healthy controls were all younger than the HD patients $(44.86 \pm 9.85$ vs. $64.97 \pm 13.20$, $p<0.001$; and $50.07 \pm 19.02$ vs. $68.51 \pm 10.35, p=0.001$, respectively) in both vaccination groups. We obtained GMTs of $10.68 \mathrm{IU} / \mathrm{mL}$ and $43.01 \mathrm{IU} / \mathrm{mL}$ for $\mathrm{nAbs}$ in serum samples from ChAdOx1-vaccinated HD patients and healthy controls $(p<0.001)$, respectively 
(Table 4, Figure 3a). The GMT values from mRNA-1273-vaccinated HD patients and healthy controls were $36.39 \mathrm{IU} / \mathrm{mL}$ and $262.2 \mathrm{IU} / \mathrm{mL}(p=0.001)$, respectively (Table 4 , Figure $3 c)$. Because of significant differences in age between HD patients and normal controls, a resampling subset of patients with individually matched ages ( \pm 2 years) as built. Again, we found that HD patients had lower GMTs for nAbs than healthy controls receiving either $\mathrm{ChAdOx1}$ (12.91 vs. $36.98 \mathrm{IU} / \mathrm{mL}, p<0.001$ ) or mRNA-1273 (60.22 vs. $248.1 \mathrm{IU} / \mathrm{mL}, p=0.038$ ) (Figure 3b,d). Specifically, healthy controls receiving mRNA-1273 had a fourfold increase in GMT values compared to HD patients. With regard to positive humoral response rate, HD patients also had a lower rate than that of healthy controls with both types of vaccine $(13.2 \%$ vs. $52.2 \%, p<0.001$; and $46.2 \%$ vs. $93.3 \%, p=0.004$, respectively) (Table 4). This finding confirms the attenuation of humoral response to a single dose of COVID-19 vaccine in uremic patients.

Table 4. Different humoral responses to two types of COVID-19 vaccine.

\begin{tabular}{|c|c|c|c|c|c|c|}
\hline & \multicolumn{2}{|c|}{ ChAdOx1n (Oxford-AstraZeneca) } & \multicolumn{4}{|c|}{ mRNA-1273 (Moderna) } \\
\hline & $\begin{array}{l}\text { HD Group } \\
(n=174)\end{array}$ & $\begin{array}{l}\text { Control Group } \\
\quad(n=67)\end{array}$ & $p$-Value & $\begin{array}{l}\text { HD Group } \\
\quad(n=26)\end{array}$ & $\begin{array}{l}\text { Control Group } \\
\qquad(n=15)\end{array}$ & $p$-Value \\
\hline Age (years) & $64.97 \pm 13.20$ & $44.86 \pm 9.85$ & $<0.001 *$ & $68.51 \pm 10.35$ & $50.07 \pm 19.02$ & $0.001 *$ \\
\hline Male, $n(\%)$ & $94(54)$ & $22(32.8)$ & $0.003 *$ & $7(26.9)$ & $13(86.7)$ & $<0.001$ * \\
\hline $\begin{array}{l}\text { Predicted } \mathrm{NT}_{50} \\
\text { titer (median, } \\
\mathrm{IQR})(\mathrm{IU} / \mathrm{mL})\end{array}$ & $6.85(5.89-10.68)$ & 40.01 (9.58-186.71) & $<0.001 *$ & $20.27(6.83-189.66)$ & 319.44 (196.54-461.48) & $0.001 *$ \\
\hline $\begin{array}{c}\text { GMTs }(95 \% \mathrm{CI}) \\
(\mathrm{IU} / \mathrm{mL})\end{array}$ & $10.68(9.10-12.54)$ & $43.01(30.11-61.42)$ & & $36.39(17.95-73.77)$ & $262.2(133.9-513.4)$ & \\
\hline $\begin{array}{c}\text { Humoral } \\
\text { response, } n(\%)\end{array}$ & $23(13.2)$ & $35(52.2)$ & $<0.001 *$ & $12(46.2)$ & $14(93.3)$ & 0.004 * \\
\hline
\end{tabular}

Notes: Data are presented as mean \pm standard deviation or median (interquartile range). Abbreviations- $\mathrm{NT}_{50}$ SARS-CoV-2 50\% neutralization titer, expressed in international units; GMTs: geometric mean titers; Humoral responses were defined as having predicted $\mathrm{NT}_{50}>35.13 \mathrm{IU} / \mathrm{mL}$. *: Statistically significant.

\subsection{Different Humoral Responses between mRNA and Vector-Based Vaccines}

The adenoviral vector vaccine ChAdOx1 elicited lower humoral response with lower GMTs for anti-S1 and anti-RBD antibodies than the mRNA-1273 vaccine in both HD patients (anti-S1: 2.74 vs. $5.26 \mathrm{EU} / \mathrm{mL}, p=0.001$; anti-RBD: 2.43 vs. $2.64 \mathrm{EU} / \mathrm{mL}, p<0.001$ ) and healthy controls (anti-S1: 5.46 vs. $19.57 \mathrm{EU} / \mathrm{mL}, p<0.001$; anti-RBD: 4.27 vs. $12.97 \mathrm{EU} / \mathrm{mL}$, $p<0.001$; Figure $4 \mathrm{a}, \mathrm{b})$. Similarly, the GMTs of predicted $\mathrm{NT}_{50}$ calculated from GAM regression analysis, using anti-S1 and anti-RBD as predictors, were significantly lower in ChAdOx1-vaccinated compared to mRNA-1273-vaccinated subjects in both the HD $(10.68$ vs. $36.39, p<0.001)$ and control groups (43.01 vs. $262.22, p<0.001$; Figure $4 c)$. The results suggest that the mRNA-based vaccine mRNA- 1273 could elicit more prominent $\mathrm{nAb}$ responses than the adenoviral vector vaccine ChAdOx1 in both HD patients and healthy controls. 
a ChAdOx1 (Oxford-AstraZeneca). All individuals

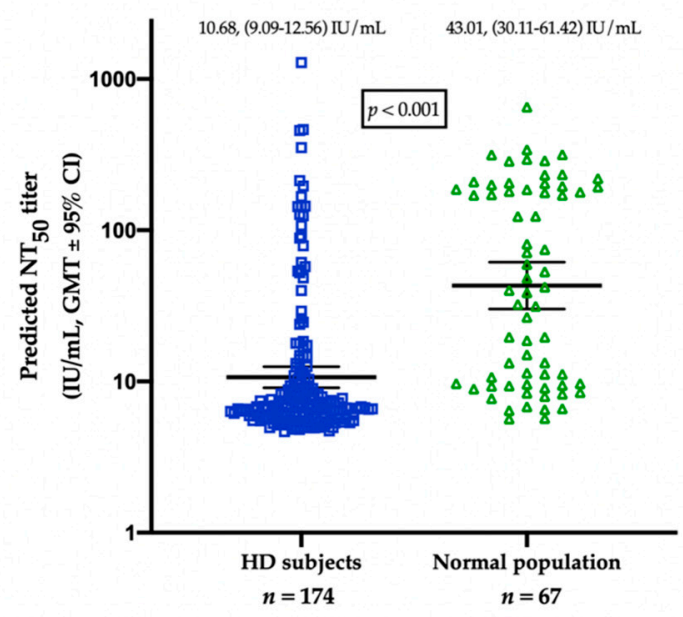

c

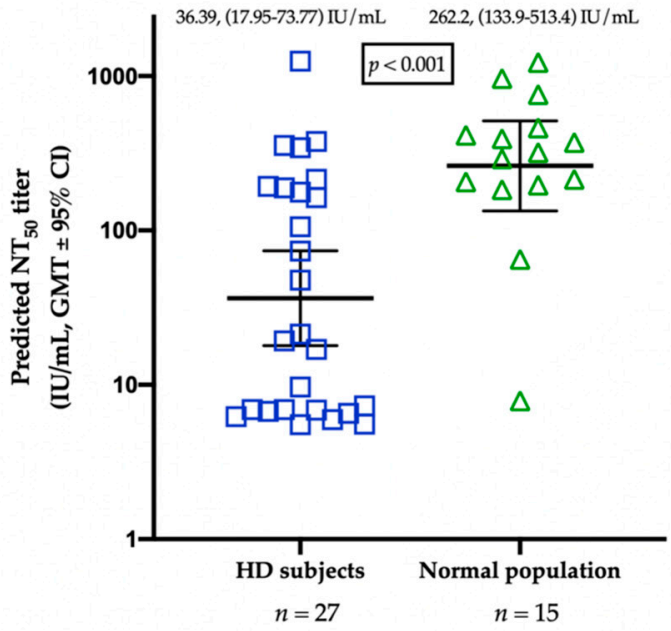

\section{b ChAdOx1 (Oxford-AstraZeneca). Age-matched}

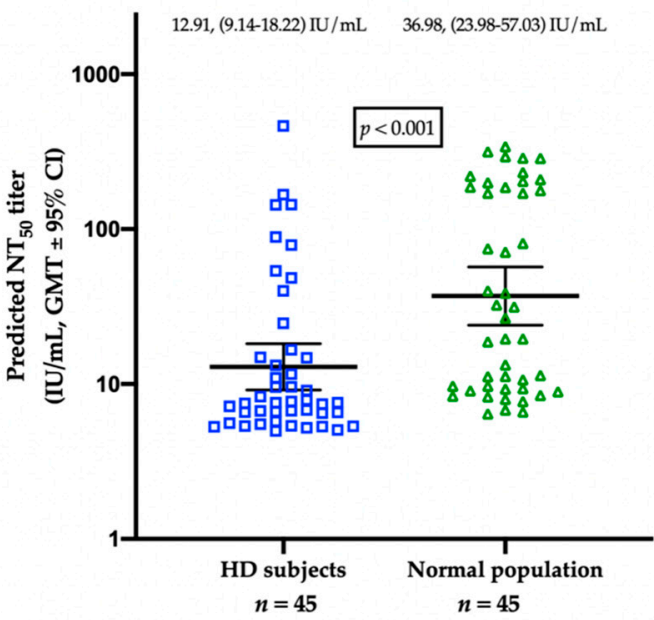

d

mRNA-1273 (Moderna). Age-matched

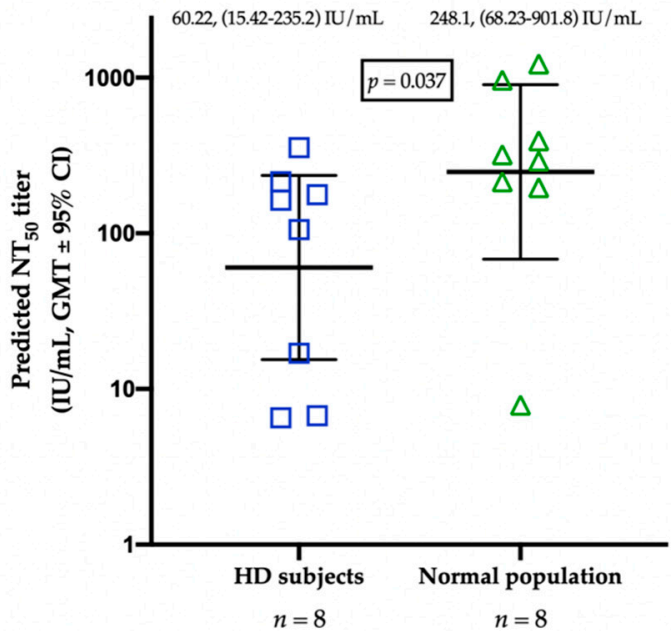

Figure 3. (a) Comparison of geometric mean titers (GMTs) for neutralizing antibodies (nAbs) between hemodialysis patients and healthy controls vaccinated with a first dose of ChAdOx1. (b) Comparison of GMTs for nAbs from age-matched hemodialysis patients and healthy controls vaccinated with a first dose of ChAdOx1. (c) Comparison of geometric mean titers (GMTs) for nAbs from hemodialysis patients and healthy controls vaccinated with a first dose of mRNA-1273. (d) Comparison of GMTs for $\mathrm{nAbs}$ from age-matched hemodialysis patients and healthy controls vaccinated with a first dose of mRNA-1273. 

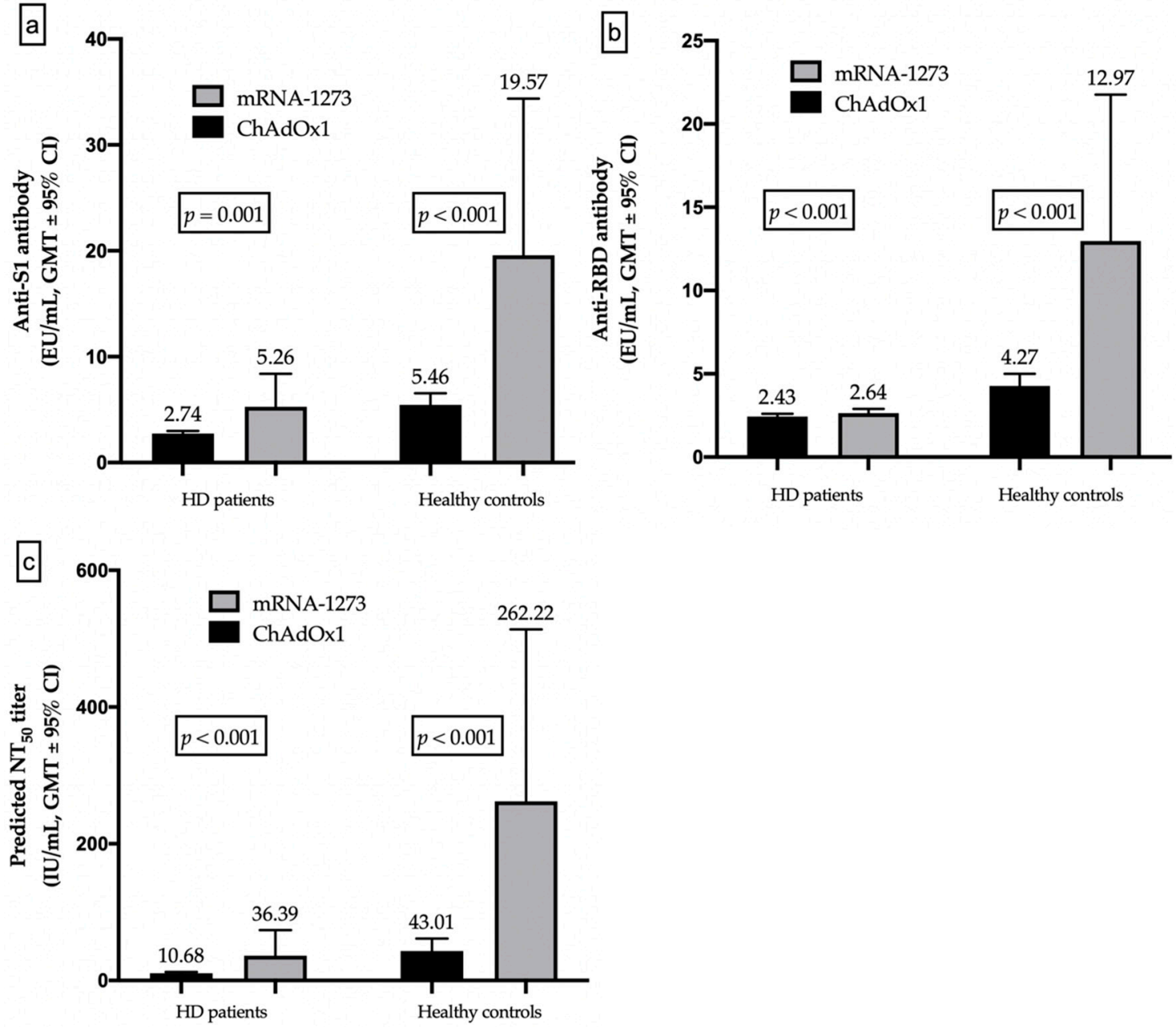

Figure 4. Comparison of humoral responses between ChAdOx1- and mRNA-1273-vaccinated individuals: (a) Geometric mean titers (GMTs) of anti-S1 antibodies; (b) GMTs of anti-receptor-bindingdomain (RBD) antibodies; (c) GMTs of predicted SARS-CoV-2 50\% neutralization titer $\left(\mathrm{NT}_{50}\right)$.

\section{Discussion}

In this prospective observational single-center study, we demonstrated that HD patients had decreased humoral responses to COVID-19 vaccination compared to healthy controls. Older age and high cardiothoracic ratio were independently associated with lower $\mathrm{NT}_{50}$ of nAbs in HD patients receiving a single dose of ChAdOx1 vaccination. The relationship remained significant after adjusting for albumin, gender, and diabetes. However, the GMTs for $n A b s$ were more prominent after a single dose of mRNA-1273 vaccination than after ChAdOx1 vaccination, in both HD patients and healthy controls.

A single dose of ChAdOx1 vaccine achieves a 76\% efficacy for symptomatic COVID-19 after 21 days post-vaccination, and an extension of the dose interval to 12 weeks might provide advantages over an interval of less than 6 weeks [27]. We obtained nAb titers from HD patients on day 56, and from healthy controls on a median of day 30 postvaccination. However, anti-spike-protein antibodies elicited by ChAdOx1 peaked on day 28 , and stayed elevated on day 56 as well as the comparable neutralization test, while Voysey et al. showed only a mild decrease of anti-spike-protein antibodies on day 56 compared with day $28[27,28]$. This phenomenon suggests that the different time of blood sampling between the two groups had a limited influence on the results of our study. In the early stage of the outbreak in mid-May 2021, Taiwan was facing serious difficulties in obtaining vaccines; the initial acquired vaccines were mainly ChAdOx1. Perhaps the results of the above-mentioned research could give Taiwan's health authorities more confidence 
to generally promote first-dose vaccination and postpone the second dose when supplies are scarce.

Many studies have evaluated mRNA vaccines in dialysis patients, but only a few studies are available on vector-based vaccines [15,16,29-32]. The findings of our study are comparable to those of several other studies, showing that the mRNA-1273 vaccine can elicit higher $\mathrm{nAb}$ titers than the ChAdOx1 vaccine in both HD patients and healthy controls. Garcia et al. conducted a study assessing the humoral response in dialysis patients by estimating total anti-RBD response, including IgG and IgM titers, and found that one-third of patients $(n=367)$ inoculated with the adenovirus Ad26.COV 2.S (Johnson \& Johnson, attenuated adenovirus) vaccine failed to seroconvert. In that cohort, $36 \%$ of vaccinated subjects had no detectable or diminished IgG response even 28-60 days postvaccination [15]. Candice et al. showed that $C h A d O x 1$ vaccination provided inferior humoral response compared to the mRNA vaccine BNT162b2 (BioNTech/Pfizer) in an HD cohort assessed by titers of anti-S antibodies $(n=272)$ [29]. Although the shortage of vaccines is a global challenge, the findings of this and other studies highlight the importance of application of effective vaccines in the most vulnerable populations, such as HD patients.

Most studies assessed humoral responses by estimating anti-S1 or anti-RBD IgG or IgM as a surrogate for $\mathrm{nAbs}$; however, these antibodies might indicate past or recent exposure to COVID-19, rather than neutralizing ability. Investigations concerning viral neutralization tests in HD cohorts remain scarce. Only a UK study applied live virus microneutralization in seronegative HD patients, comparing ChAdOx1 $(n=53)$ and BNT162b2 $(n=55)$ response, and found that the mRNA vaccine provokes comparable nAb titers in HD patients and healthy controls, while ChAdOx1 induces suboptimal $\mathrm{nAb}$ titers against all variants of concern (VOCs), including the delta variant that is dominant worldwide [16]. Our study used ELISA procedures to detect concentrations of both anti-S1 and anti-RBD antibodies, including IgG, IgM, and IgA, and the results were computed by a spline-based two-variable GAM to approximate real $\mathrm{NT}_{50}$ titers for $\mathrm{nAbs}$; the correlation between predicted and actual virus neutralization titers was as high as 0.917 , according to the manufacturer. Real $\mathrm{NT}_{50}$ (IU/mL) from a biosafety level 3 laboratory was used as a standard to assess whether these widely used commercial serological assays reflect the neutralization titers based on the detection of antibodies against S1 and/or RBD. The highest correlation was observed between the titers obtained by the MeDiPro and the live SARS-CoV-2 neutralization test assays $(r=0.9111)$, which were superior to widely used commercial assays such as the Roche and Abbott RBD antibody titers, with fair correlation coefficients of 0.7294 and 0.8466 , respectively [33]. Accordingly, this method might serve as a surrogate for the rapid quantification of $\mathrm{nAb}$ titers, replacing the conventional laboratories and time-consuming virus neutralization tests. Therefore, it represents a safer, quicker, and more efficient way to quantify nAbs than other tests. To our knowledge, our study is the first attempt to explore using the predicted $\mathrm{NT}_{50}$ for quantifying the $\mathrm{nAb}$ response in an extensive cohort of HD patients $(n=200)$ vaccinated with ChAdOx1.

The definition of positive humoral response rate used for our laboratory method is the predicted $\mathrm{NT}_{50} \geq 35.13 \mathrm{IU} / \mathrm{mL}$, which is more stringent than previous studies using occurrence of $\operatorname{IgG}$ or $\operatorname{IgM}$ as indicators of seroconversion. This may in part underestimate the response rate of our HD patients compared to the levels of previous studies. Moreover, the response rate was indeed lower in HD patients than in healthy controls, especially in the ChAdOx1 group (13.2\%). Uremic patients have defects in innate and adaptive immunity [34]; in the uremic milieu, antigen-presenting cell dysfunction and memory T-cell apoptosis may reduce antibody production by B cells. In addition, signals of B-cell differentiation are also damaged [35-37]. The impairment of antigen-presenting capability further makes the immune systems of uremic patients struggle to recognize pathogens and to produce a series of downstream adaptive immunity [38,39]. Though humoral immunity cannot be representative of overall immunity, and the ChAdOx1 vaccine was reported to elicit more prominent cellular immunity than mRNA vaccines, the lack of an $n A b$ response was a strong predictor of death, adjusted for age and gender [40-42]. This phenomenon 
suggests that immunocompromised subjects-including those with uremia, solid organ transplants, or malignancy-might receive a heterologous vaccination strategy with an mRNA vaccine as a second dose to boost serum $n A b$ quantity, if primed with ChAdOx1 as the first dose.

Diabetes, albumin, C-reactive protein, and Kt/V were important indicators of clinical outcomes in HD patients; however, these parameters were not correlated with levels of $\mathrm{nAb}$ production in our study. Our findings were similar to those of a study on Mexico's healthcare workers, showing that gender and comorbidities (such as diabetes, obesity, and hypertension) are not associated with low titers of nAbs [43,44]. A relatively homogeneous status regarding nutrition, inflammation, and administered dialysis dosage may all contribute to this phenomenon.

Cardiothoracic ratio is one of the monitoring indicators of dialysis quality, and is usually used to assess the fluid status of dialysis patients, and as an important reference for dry weight adjustment. Cardiothoracic ratio has been found to be positively associated with C-reactive protein and inversely associated with albumin, and can predict 2-year all-cause mortality and infection-caused mortality in HD populations [45-47]. High cardiothoracic ratio is also reported to be a significant and independent predictor of cardiovascular events in patients undergoing HD [48-50]. Cardiovascular disease has been viewed as a risk factor for severe COVID-19, and is strongly associated with in-hospital death [51,52]. High cardiothoracic ratio has been linked to chronic inflammation and malnutrition in HD patients, and the status of chronic inflammation might hinder the production of nAbs [53]. This phenomenon possibly illustrates the connection between high cardiothoracic ratio and low $\mathrm{nAb}$ titers found in our study.

This study has some limitations. First, a single dose of COVID-19 vaccination could not precisely predict the final results after full vaccination-it simply reflects the humoral response during that period when Taiwan faced a shortage of vaccines, and even the prioritized, vulnerable patients only received a single dose. Second, we did not measure $\mathrm{nAbs}$ before vaccination. There was almost no domestic transmission before May 2021 in Taiwan, and there were no confirmed cases in our HD center, in combination with weekly rapid antigen testing on our HD patients in case of asymptomatic infection; hence, the patients were supposed to be negative for nAbs. Third, we used a relatively small sample, and we only performed further analyses in the ChAdOx1 group to explore potential factors associated with responses to vaccination. The mRNA-1273 group and control group enrolled fewer people, and the subjects in the HD cohort and healthy controls were highly heterogeneous in terms of age and sex. Hence, we built a resampling subset of patients individually matched for age, which yielded similar results. Nevertheless, to our knowledge, we used the largest number of HD patients receiving ChAdOx1 enrolled for $\mathrm{NT}_{50}$ analyses compared with previous studies. Fourth, we did not conduct baseline laboratory testing of healthy controls, because they were generally healthy, and we simply collected blood samples for measurement of nAbs. Finally, $\mathrm{NT}_{50}$ titers were not predicted through real neutralization testing in our study; however, the assay we used has been proven to have better correlation with real neutralization tests than other commercial serological tests [33].

\section{Conclusions}

This prospective, observational, single-center study established the associations of high cardiothoracic ratio and old age with reduced $\mathrm{nAb}$ titers in an HD cohort vaccinated with a single dose of ChAdOx1. The GMTs for $n A b$ s were lower in HD patients vaccinated with ChAdOx1 or mRNA-1273. mRNA-1273 could elicit higher $n$ Abs and positive humoral response rate in both $\mathrm{HD}$ patients and healthy controls.

We also noticed that a single dose of ChAdOx1 only provided $13.2 \%$ of HD patients with sufficient nAbs, while mRNA-1273 provided close to half of HD patients with a positive humoral response. To enhance dialysis quality and avoid severe COVID-19, maintaining adequate cardiothoracic ratio by close monitoring of fluid status is worthy of 
our scrutiny, and we should also consider offering an mRNA vaccine as a second dose to boost $\mathrm{nAb}$ quantity if patients were vaccinated with $\mathrm{ChAdOx} 1$ as a first dose.

Supplementary Materials: The following supporting information can be downloaded at https://ww w.mdpi.com/article/10.3390/jpm12010068/s1: Table S1: Comparisons of demographic and clinical characteristics between enrolled and non-enrolled patients on hemodialysis; Table S2: Comparisons of demographic and clinical characteristics between ChAdOx1- (Oxford-AstraZeneca) and mRNA-1273 (Moderna)-vaccinated patients on hemodialysis; Figure S1: Correlation between the age and predicted SARS-CoV-2 50\% neutralization titer $\left(\mathrm{NT}_{50}\right)$ in the ChAdOx1 group (Panel a) and mRNA-1273 group (Panel b).

Author Contributions: C.-Y.C.: writing-original draft, formal analysis, project administration, resources; K.-T.L.: software, methodology, data curation, validation; S.-R.S.: conceptualization, visualization, resources, supervision; J.-J.Y., Y.-T.C., H.-C.P., H.-J.H., C.-Y.S. and C.-C.L. (Chin-Chan Lee): investigations; C.-Y.W.: investigations, methodology; C.-C.L. (Chi-Chun Lai): visualization, supervision; I.-W.W.: supervision, conceptualization, writing-review and editing, project administration, funding acquisition. All authors have read and agreed to the published version of the manuscript.

Funding: This research was funded by research grants from the Chang Gung Memorial Hospital (CMRPG2L0231) and the National Institutes of Health USA grant U01 AI151698 for the United World Arbovirus Research Network (UWARN).

Institutional Review Board Statement: This study was performed in accordance with the Declaration of Helsinki, and was approved by the Ethics Committee of the Institutional Review Board at Chang Gung Medical Foundation. The approval number is 202001041A3C604 and 202100854B0A3.

Informed Consent Statement: Informed consent was obtained from all subjects involved in the study.

Data Availability Statement: Not applicable.

Acknowledgments: We wish to express our deepest gratitude to all of the people who participated in this study. We also express our gratitude to all of the nephrology medical and nursing staff members for their dedicated efforts during the COVID-19 epidemic; to the Research Center for Emerging Viral Infections, for diligent, excellent, and rapid quantification of neutralizing antibodies; to the Committee of Infection Control, for suggestions of infection prevention; and to the Department of Administration, for excellent support in terms of medical equipment, manpower, and resource allocation.

Conflicts of Interest: The authors declare no conflict of interest.

\section{References}

1. WHO. Coronavirus Disease (COVID-19) Dashboard. Available online: https:/ / covid19.who.int (accessed on 5 December 2021).

2. McMichael, T.M.; Currie, D.W.; Clark, S.; Pogosjans, S.; Kay, M.; Schwartz, N.G.; Lewis, J.; Baer, A.; Kawakami, V.; Lukoff, M.D.; et al. Epidemiology of COVID-19 in a Long-Term Care Facility in King County, Washington. N. Engl. J. Med. 2020, 382, 2005-2011. [CrossRef] [PubMed]

3. Zhou, F.; Yu, T.; Du, R.; Fan, G.; Liu, Y.; Liu, Z.; Xiang, J.; Wang, Y.; Song, B.; Gu, X.; et al. Clinical course and risk factors for mortality of adult inpatients with COVID-19 in Wuhan, China: A retrospective cohort study. Lancet 2020, 395, 1054-1062. [CrossRef]

4. CDC COVID-19 Response Team; Chow, N.; Fleming-Dutra, K.; Gierke, R.; Hall, A.; Hughes, M. Preliminary estimates of the prevalence of selected underlying health conditions among patients with coronavirus disease 2019-United States, February 12-March 28, 2020. Morb. Mortal. Wkly. Rep. 2020, 69, 382.

5. Rao, K.S.; Suryaprakash, V.; Senthilkumar, R.; Preethy, S.; Katoh, S.; Ikewaki, N.; Abraham, S.J.K. Role of Immune Dysregulation in Increased Mortality among a Specific Subset of COVID-19 Patients and Immune-Enhancement Strategies for Combatting through Nutritional Supplements. Front. Immunol. 2020, 11, 1548. [CrossRef]

6. Taiwan End Stage Renal Disease Registry. 2020 Annual Report on Kidney Disease in Taiwan. Available online: https://www.tsn. org.tw/UI/L/TWRD/ebook_2020\%E5\%B9\%B4\%E5\%A0\%B1.pdf (accessed on 1 January 2022).

7. Bregman, H.D.J.; Ing, T.S. Complications during hemodialysis. In Handbook of Dialysis, 2nd ed.; Dauugirdas, J.T., Ing, T.S., Eds.; Lippincott Williams and Wilkins: New York, NY, USA, 1994; p. 149.

8. Liu, Y.F.; Zhang, Z.; Pan, X.L.; Xing, G.L.; Zhang, Y.; Liu, Z.S.; Tu, S.H. The chronic kidney disease and acute kidney injury involvement in COVID-19 pandemic: A systematic review and meta-analysis. PLoS ONE 2021, 16, e0244779. [CrossRef]

9. Betjes, M.G. Immune cell dysfunction and inflammation in end-stage renal disease. Nat. Rev. Nephrol. 2013, 9, 255-265. [CrossRef] [PubMed] 
10. Corbett, R.W.; Blakey, S.; Nitsch, D.; Loucaidou, M.; McLean, A.; Duncan, N.; Ashby, D.R.; West London Renal and Transplant Centre. Epidemiology of COVID-19 in an Urban Dialysis Center. J. Am. Soc. Nephrol. 2020, 31, 1815-1823. [CrossRef]

11. Chen, C.Y.; Shao, S.C.; Chen, Y.T.; Hsu, C.K.; Hsu, H.J.; Lee, C.C.; Sun, C.Y.; Chen, Y.C.; Hung, M.J.; Wu, I.W. Incidence and Clinical Impacts of COVID-19 Infection in Patients with Hemodialysis: Systematic Review and Meta-Analysis of 396,062 Hemodialysis Patients. Healthcare 2021, 9, 47. [CrossRef]

12. Chodick, G.; Tene, L.; Rotem, R.S.; Patalon, T.; Gazit, S.; Ben-Tov, A.; Weil, C.; Goldshtein, I.; Twig, G.; Cohen, D.; et al. The effectiveness of the TWO-DOSE BNT162b2 vaccine: Analysis of real-world data. Clin. Infect. Dis. 2021. [CrossRef]

13. Tenforde, M.W.; Patel, M.M.; Ginde, A.A.; Douin, D.J.; Talbot, H.K.; Casey, J.D.; Mohr, N.M.; Zepeski, A.; Gaglani, M.; McNeal, T.; et al. Effectiveness of SARS-CoV-2 mRNA Vaccines for Preventing COVID-19 Hospitalizations in the United States. Clin. Infect. Dis. 2021. [CrossRef]

14. Boyarsky, B.J.; Werbel, W.A.; Avery, R.K.; Tobian, A.A.R.; Massie, A.B.; Segev, D.L.; Garonzik-Wang, J.M. Antibody Response to 2-Dose SARS-CoV-2 mRNA Vaccine Series in Solid Organ Transplant Recipients. JAMA 2021, 325, 2204-2206. [CrossRef]

15. Garcia, P.; Anand, S.; Han, J.; Montez-Rath, M.E.; Sun, S.; Shang, T.; Parsonnet, J.; Chertow, G.M.; Schiller, B.; Abra, G. COVID-19 Vaccine Type and Humoral Immune Response in Patients Receiving Dialysis. J. Am. Soc. Nephrol. 2022, 33, 33-37. [CrossRef] [PubMed]

16. Carr, E.J.; Wu, M.; Harvey, R.; Wall, E.C.; Kelly, G.; Hussain, S.; Howell, M.; Kassiotis, G.; Swanton, C.; Gandhi, S.; et al Neutralising antibodies after COVID-19 vaccination in UK haemodialysis patients. Lancet 2021, 398, 1038-1041. [CrossRef]

17. Danthu, C.; Hantz, S.; Dahlem, A.; Duval, M.; Ba, B.; Guibbert, M.; El Ouafi, Z.; Ponsard, S.; Berrahal, I.; Achard, J.M.; et al. Humoral Response after SARS-CoV-2 mRNA Vaccination in a Cohort of Hemodialysis Patients and Kidney Transplant Recipients J. Am. Soc. Nephrol. 2021, 32, 2153-2158. [CrossRef]

18. Speer, C.; Goth, D.; Benning, L.; Buylaert, M.; Schaier, M.; Grenz, J.; Nusshag, C.; Kalble, F.; Kreysing, M.; Reichel, P.; et al. Early Humoral Responses of Hemodialysis Patients after COVID-19 Vaccination with BNT162b2. Clin. J. Am. Soc. Nephrol. 2021, 16, 1073-1082. [CrossRef] [PubMed]

19. Taiwan Centers for Disease Control. COVID-19 Statistics of Taiwan. Available online: https://sites.google.com/cdc.gov.tw/2019 ncov/taiwan?authuser $=0$ (accessed on 5 December 2021).

20. Chen, C.Y.; Ye, J.J.; Huang, T.S.; Lee, C.C.; Chen, Y.T.; Hsu, C.K.; Hsu, H.J.; Sun, C.Y.; Pan, H.C.; Chen, K.S.; et al. Effective Preventive Strategies to Prevent Secondary Transmission of COVID-19 in Hemodialysis Unit: The First Month of Community Outbreak in Taiwan. Healthcare 2021, 9, 1173. [CrossRef] [PubMed]

21. Francis, A.; Baigent, C.; Ikizler, T.A.; Cockwell, P.; Jha, V. The urgent need to vaccinate dialysis patients against severe acute respiratory syndrome coronavirus 2: A call to action. Kidney Int. 2021, 99, 791-793. [CrossRef] [PubMed]

22. American Society of Nephrology. Prioritizing COVID-19 Vaccination in Dialysis. Available online: https://www.kidn eynews.org/view/news/policy-advocacy/leading-edge/prioritizing-COVID-19-vaccination-in-dialysis.xml (accessed on 24 November 2021).

23. Combe, C.; Kirsch, A.H.; Alfano, G.; Luyckx, V.A.; Shroff, R.; Kanbay, M.; van der Sande, F.; Basile, C.; The EUDIAL Working Group of the ERA-EDTA. At least 156 reasons to prioritize COVID-19 vaccination in patients receiving in-centre haemodialysis Nephrol. Dial. Transplant. 2021, 36, 571-574. [CrossRef]

24. Taiwan Society of Nephrology. Taiwan Society of Nephrology. Statement from the Taiwan Society of Nephrology: Regarding the Administration of COVID-19 503 Vaccines for Dialysis Facilities and Renal Patients. Available online: https:/ / www.tsn.org.tw/tsnFile/authority / F8D9162FFC199F7B/\%E5\%8F\%B0\%E7\%81\%A3\%E8\%85\%8E \%E8\%87\%9F\%E

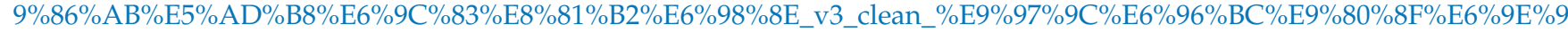
0\%E9\%99\%A2\%E6\%89\%80\%E5\%8F\%8A\%E8\%85\%8E\%E8\%87\%9F\%E7\%97\%85\%E4\%BA \%BACOVID-19\%E7\%96\%AB \%E8\%8 B\%97\%E6\%96\%BD\%E6\%89\%93_1100513.pdf (accessed on 24 November 2021).

25. Singh, A.K.; Singh, A.; Singh, R.; Misra, A. Molnupiravir in COVID-19: A systematic review of literature. Diabetes Metab. Syndr. Clin. Res. Rev. 2021, 15, 102329. [CrossRef]

26. United States Renal Data System. 2020 USRDS Annual Data Report: Incidence, Prevalence, Patient Characteristics, and Treatment Modalities. Available online: https:/ / adr.usrds.org/2020/end-stage-renal-disease/1-incidence-prevalence-patient-characteri stics-and-treatment-modalities (accessed on 24 November 2021).

27. Voysey, M.; Clemens, S.A.C.; Madhi, S.A.; Weckx, L.Y.; Folegatti, P.M.; Aley, P.K.; Angus, B.; Baillie, V.L.; Barnabas, S.L.; Bhorat, Q.E.; et al. Single-dose administration and the influence of the timing of the booster dose on immunogenicity and efficacy of ChAdOx1 nCoV-19 (AZD1222) vaccine: A pooled analysis of four randomised trials. Lancet 2021, 397, 881-891. [CrossRef]

28. Folegatti, P.M.; Ewer, K.J.; Aley, P.K.; Angus, B.; Becker, S.; Belij-Rammerstorfer, S.; Bellamy, D.; Bibi, S.; Bittaye, M.; Clutterbuck, E.A.; et al. Safety and immunogenicity of the ChAdOx1 nCoV-19 vaccine against SARS-CoV-2: A preliminary report of a phase 1/2, single-blind, randomised controlled trial. Lancet 2020, 396, 467-478. [CrossRef]

29. Clarke, C.L.; Martin, P.; Gleeson, S.; Thomson, T.; Edwards, H.; Mortimer, P.; McIntyre, S.; Deborah, J.; Cox, A.; Pickard, G.; et al. Comparison of immunogenicity between BNT162b2 and ChAdOx1 SARS-CoV-2 vaccines in a large haemodialysis population. medRxiv 2021. [CrossRef]

30. Billany, R.E.; Selvaskandan, H.; Adenwalla, S.F.; Hull, K.L.; March, D.S.; Burton, J.O.; Bishop, N.C.; Carr, E.J.; Beale, R.; Tang, J.W.; et al. Seroprevalence of antibody to S1 spike protein following vaccination against COVID-19 in patients receiving hemodialysis: A call to arms. Kidney Int. 2021, 99, 1492-1494. [CrossRef] [PubMed] 
31. Lesny, P.; Anderson, M.; Cloherty, G.; Stec, M.; Haase-Fielitz, A.; Haarhaus, M.; Santos, C.; Lucas, C.; Macario, F.; Haase, M. Immunogenicity of a first dose of mRNA- or vector-based SARS-CoV-2 vaccination in dialysis patients: A multicenter prospective observational pilot study. J. Nephrol. 2021, 34, 975-983. [CrossRef]

32. Anand, S.; Montez-Rath, M.E.; Han, J.; Garcia, P.; Cadden, L.; Hunsader, P.; Kerschmann, R.; Beyer, P.; Dittrich, M.; Block, G.A.; et al. Antibody Response to COVID-19 Vaccination in Patients Receiving Dialysis. J. Am. Soc. Nephrol. 2021, 32, 2435-2438. [CrossRef] [PubMed]

33. Kung, Y.A.; Huang, C.G.; Huang, S.Y.; Liu, K.T.; Huang, P.N.; Yu, K.Y.; Yang, S.L.; Chen, C.P.; Cheng, C.Y.; Lin, Y.T.; et al. Antibody titers measured by commercial assays are correlated with neutralizing antibody titers calibrated by international standards. medRxiv 2021. [CrossRef]

34. Sharif, M.R.; Chitsazian, Z.; Moosavian, M.; Raygan, F.; Nikoueinejad, H.; Sharif, A.R.; Einollahi, B. Immune disorders in hemodialysis patients. Iran. J. Kidney Dis. 2015, 9, 84-96.

35. Eleftheriadis, T.; Antoniadi, G.; Liakopoulos, V.; Kartsios, C.; Stefanidis, I. Basic Science and Dialysis: Disturbances of acquired immunity in hemodialysis patients. Semin. Dial. 2007, 20, 440-451. [CrossRef] [PubMed]

36. Syed-Ahmed, M.; Narayanan, M. Immune Dysfunction and Risk of Infection in Chronic Kidney Disease. Adv. Chronic Kidney Dis. 2019, 26, 8-15. [CrossRef]

37. Pahl, M.V.; Gollapudi, S.; Sepassi, L.; Gollapudi, P.; Elahimehr, R.; Vaziri, N.D. Effect of end-stage renal disease on B-lymphocyte subpopulations, IL-7, BAFF and BAFF receptor expression. Nephrol. Dial. Transplant. 2010, 25, 205-212. [CrossRef] [PubMed]

38. Hou, Y.C.; Lu, K.C.; Kuo, K.L. The Efficacy of COVID-19 Vaccines in Chronic Kidney Disease and Kidney Transplantation Patients: A Narrative Review. Vaccines 2021, 9, 885. [CrossRef]

39. Kim, K.W.; Chung, B.H.; Jeon, E.J.; Kim, B.M.; Choi, B.S.; Park, C.W.; Kim, Y.S.; Cho, S.G.; Cho, M.L.; Yang, C.W. B cell-associated immune profiles in patients with end-stage renal disease (ESRD). Exp. Mol. Med. 2012, 44, 465-472. [CrossRef]

40. Dispinseri, S.; Secchi, M.; Pirillo, M.F.; Tolazzi, M.; Borghi, M.; Brigatti, C.; De Angelis, M.L.; Baratella, M.; Bazzigaluppi, E.; Venturi, G.; et al. Neutralizing antibody responses to SARS-CoV-2 in symptomatic COVID-19 is persistent and critical for survival. Nat. Commun. 2021, 12, 2670. [CrossRef]

41. Parry, H.; Bruton, R.; Stephens, C.; Brown, K.; Amirthalingam, G.; Otter, A.; Hallis, B.; Zuo, J.; Moss, P. Differential immunogenicity of BNT162b2 or ChAdOx1 vaccines after extended-interval homologous dual vaccination in older people. Immun. Ageing 2021, 18, 34. [CrossRef]

42. Parry, H.; Bruton, R.; Tut, G.; Ali, M.; Stephens, C.; Greenwood, D.; Faustini, S.; Hughes, S.; Huissoon, A.; Meade, R.; et al Immunogenicity of single vaccination with BNT162b2 or ChAdOx1 nCoV-19 at 5-6 weeks post vaccine in participants aged 80 years or older: An exploratory analysis. Lancet Healthy Longev. 2021, 2, e554-e560. [CrossRef]

43. Morales-Núñez, J.J.; Muñoz-Valle, J.F.; Torres-Hernández, P.C.; Hernández-Bello, J. Overview of Neutralizing Antibodies and Their Potential in COVID-19. Vaccines 2021, 9, 1376. [CrossRef]

44. Morales-Nunez, J.J.; Munoz-Valle, J.F.; Meza-Lopez, C.; Wang, L.F.; Machado Sulbaran, A.C.; Torres-Hernandez, P.C.; Bedolla-Barajas, M.; la O-Gómez, D.; Balcazar-Felix, P.; Hernandez-Bello, J. Neutralizing Antibodies Titers and Side Effects in Response to BNT162b2 Vaccine in Healthcare Workers with and without Prior SARS-CoV-2 Infection. Vaccines 2021, 9, 742. [CrossRef]

45. Chen, K.H.; Lin-Tan, D.T.; Huang, W.H.; Hung, C.C.; Chang, C.T.; Huang, J.Y.; Lin, J.L. Cardiothoracic ratio, malnutrition, inflammation, and two-year mortality in non-diabetic patients on maintenance hemodialysis. Kidney Blood Press. Res. 2008, 31, 143-151. [CrossRef]

46. Yen, T.H.; Lin, J.L.; Lin-Tan, D.T.; Hsu, K.H. Cardiothoracic ratio, inflammation, malnutrition, and mortality in diabetes patients on maintenance hemodialysis. Am. J. Med. Sci. 2009, 337, 421-428. [CrossRef]

47. Ito, K.; Ookawara, S.; Ueda, Y.; Miyazawa, H.; Yamada, H.; Goto, S.; Ishii, H.; Shindo, M.; Kitano, T.; Hirai, K.; et al. A Higher Cardiothoracic Ratio Is Associated with 2-Year Mortality after Hemodialysis Initiation. Nephron Extra 2015, 5, 100-110. [CrossRef]

48. Yotsueda, R.; Taniguchi, M.; Tanaka, S.; Eriguchi, M.; Fujisaki, K.; Torisu, K.; Masutani, K.; Hirakata, H.; Kitazono, T.; Tsuruya, K. Cardiothoracic Ratio and All-Cause Mortality and Cardiovascular Disease Events in Hemodialysis Patients: The Q-Cohort Study. Am. J. Kidney Dis. 2017, 70, 84-92. [CrossRef]

49. Chung, T.L.; Liu, Y.H.; Huang, J.C.; Wu, P.Y.; Tu, H.P.; Chen, S.C.; Chang, J.M. Prognostic Implication of Longitudinal Changes in Cardiothoracic Ratio and Aortic Arch Calcification in Hemodialysis Patients. J. Pers. Med. 2021, 11, 788. [CrossRef] [PubMed]

50. Ou, S.H.; Liu, Y.H.; Chung, T.L.; Huang, J.C.; Wu, P.Y.; Su, H.M.; Chen, S.C. Aortic Arch Calcification and Cardiomegaly Are Associated with Overall and Cardiovascular Mortality in Hemodialysis Patients. J. Pers. Med. 2021, 11, 657. [CrossRef]

51. Docherty, A.B.; Harrison, E.M.; Green, C.A.; Hardwick, H.E.; Pius, R.; Norman, L.; Holden, K.A.; Read, J.M.; Dondelinger, F.; Carson, G.; et al. Features of 20133 UK patients in hospital with COVID-19 using the ISARIC WHO Clinical Characterisation Protocol: Prospective observational cohort study. BMJ 2020, 369, m1985. [CrossRef] [PubMed]

52. Stokes, E.K.; Zambrano, L.D.; Anderson, K.N.; Marder, E.P.; Raz, K.M.; El Burai Felix, S.; Tie, Y.; Fullerton, K.E. Coronavirus Disease 2019 Case Surveillance-United States, January 22-May 30, 2020. MMWR Morb. Mortal. Wkly. Rep. 2020, 69, 759-765. [CrossRef] [PubMed]

53. Zimmermann, P.; Curtis, N. Factors That Influence the Immune Response to Vaccination. Clin. Microbiol. Rev. 2019, 32, e00084-18. [CrossRef] [PubMed] 